\title{
1D Inversion of vertical electrical sounding (VES) using a Whale Optimization Algorithm (WOA).
}

\author{
Jorge L. Abril ${ }^{1}$, Cassiano A. Bortolozo ${ }^{2}$, Francisco Marcio Barboza ${ }^{3}$, Marcos. Vasconcelos ${ }^{4}$
}

$1,{ }^{4} \mathrm{CPGG} / \mathrm{UFBA},{ }^{2} \mathrm{CEM} A D E N,{ }^{3} \mathrm{CERES}$

Copyright 2021, SBGf - Sociedade Brasileira de Geofísica

This paper was prepared for presentation during the $17^{\text {th }}$ International Congress of the Brazilian Geophysical Society held in Rio de Janeiro, Brazil, 16-19 August 2021.

Contents of this paper were reviewed by the Technical Committee of the $17^{\text {th }}$ International Congress of the Brazilian Geophysical Society and do not necessarily represent any position of the SBGf, its officers or members. Electronic reproduction or storage of any part of this paper for commercial purposes without the written consent of the Brazilian Geophysical Society is prohibited.

\section{Abstract}

Direct current resistivity surveys have been widely used in mining, geotechnical and hydrogeological studies due to the low cost and accuracy in mapping and detecting structures in subsurface. In the last two decades, global optimization methods are becoming more popular in geophysics because they do not require gradient information and no initial model to obtain a good result to infer characteristics of the subsurface resistivity distribution. In this research, we implemented a Whale Optimization Algorithm (WOA) that mimics the social behavior of the humpback whales. To demonstrate the robustness of the WOA, we test under three synthetic models with $2 \%$ of Gaussian noise. The results shows an efficient inversion process and a low uncertainty of inverted estimated model.

\section{Introduction}

A wide quantity of optimization problems in geophysics are not linear, multidimensional and characterized by complex objective functions with several local minimum. There are two techniques for solving these inverse problems: deterministic methods based on gradient of the objective function and stochastic methods, which perform a random direct search in the solution space. These last, show a high demand of computational cost when the forward modelling is expensive, also they can be trapped in false global minimum, principally, in cases where it presents a huge quantity of parameters in the model. Fortunately, 1D forward modelling of resistivity is cheap computationally and is a good candidate to apply this kind of techniques.

Different studies reported in the literature to solve problems using a linear approach (e.g., Menke 1984; Tarantola 1987). These simple techniques require an initial solution close to the true model for obtaining a good convergence. Other techniques used by solving non-linear optimization approaches have been testes (e.g., Sen et al., 1993; Chunduru et al., 1995). These non-linear approaches can find a satisfactory solution in a large search space, but they consume a lot of time. Within these global optimization methods (GOM), can be selected in three main categories: evolution based physics-based and swarm-based methods. The most popular evolution-inspired technique is Genetic Algorithms (GA) (e.g., Goldberg, 1989). Physics- based methods imitate the physical rules in the universe. The most popular algorithms are Simulated Annealing (SA), (e.g., Kirkpatrick, 1983).

The third group of nature-inspired methods includes swarm- based techniques that mimic the social behavior of groups of animals. The most popular algorithm is Particle Swarm Optimization, originally developed by (Kennedy and Eberhart 1995). This study describes a new metaheuristic optimization algorithm (namely, Whale Optimization Algorithm, WOA) mimicking the hunting behavior of humpback whales, described by (Mirjalili, 2016). This algorithm describes a technique of spiral to simulate bubble-net attacking mechanism of humpback whales. The efficiency of the WOA algorithm developed in this expanded abstract is tested by solving two synthetic models and a set of field data. Optimization results demonstrate that WOA is very competitive compared to the state of the art optimization methods.

\section{D forward modelling resistivity}

To solve the 1D forward modelling of resistivity we adopt the linear filtering method developed by (Ghost 1971). This method solves the resistivity function integral by a convolution. The corresponding apparent resistivity $\rho_{a}(d, m)$ is calculated by Koefoed, (1970), as follows in the equation (1) :

$$
\begin{aligned}
\rho_{a}(\mathrm{~d}, \mathrm{~m})=\rho_{1}+d^{2} & \int_{0}^{\infty}\left(H_{1}(\lambda, m)\right. \\
& \left.-\rho_{1}\right) \cdot J_{1}(\lambda d) \lambda d \lambda
\end{aligned}
$$

Where $d$ is the distance between current electrodes, $J_{1}$ is the first Bessel function, $\mathrm{H}_{1}$ is the resistivity transform kernel for the first layer and $\lambda$ is the integration variable. The resistivity kernel is calculated by the recurrence formula from bottom to the surface, following the equation (2) and equation (3) :

$$
\begin{gathered}
H_{n}(\lambda)=\rho_{n} \\
H_{i}(\lambda)=\left(H_{i+1}+\rho_{i} \cdot \operatorname{th}\left(\lambda h_{i}\right)\right) /\left(+H_{i+1} \cdot \operatorname{th}\left(\lambda h_{i}\right)\right. \\
\left./ \rho_{i}\right)
\end{gathered}
$$

where $H_{i}(\lambda)$ is the transform resistivity for the ith layer, th $\left(\lambda h_{i}\right)$ is the hyperbolic tangent function. This recursive formula goes upward until $\mathrm{H}_{1}$ is determinated for the surface layer. 


\section{Method}

The inverse theory is the process to obtain from a set of observations the model that produced the observations. In other words, the observations of field or collected data give a set of data vector $\mathbf{d}=\left[\mathrm{d}_{1}, \mathrm{~d}_{2}, \mathrm{~d}_{3} \ldots \mathrm{d}_{\mathrm{n}}\right]^{\top}$, which corresponds to a $\mathbf{m}$ model vector, $\mathbf{m}=\left[m_{1}, m_{2}, m_{3} \ldots m_{n}\right]^{\top}$. These vectors are related through the operator $\mathbf{g}$, in the following equation (4):

$$
\mathbf{d}=\mathrm{g}(\mathbf{m})
$$

In the inversion process, we estimate a model $\mathbf{m}_{\text {est }}$ that minimizes the cost function, which is a measure of discrepancy between the observed and computed data sets. In this abstract, we use the normalized root square mean error (NRMS), as depicted in the equation (5)

$$
\mathrm{NRMS}=\sqrt{\frac{1}{\mathrm{~N}} * \sum_{\mathrm{i}}^{\mathrm{N}} \frac{d_{o b s}(\mathrm{i})^{2}-d_{c a l}(\mathrm{i})^{2}}{d_{o b s}(\mathrm{i})^{2}}}
$$

Where $d_{\text {obs }}(\mathrm{i})$ and $\mathrm{d}_{\mathrm{cal}}(\mathrm{i})$ are observed and calculated data, respectively, and $\mathrm{N}$ is the total number of data. For both cases synthetic and real data we calculated the normalized model NRMS misfit using the above equation.

\section{Whale optimization Algorithm (WOA)}

Bases on the social behavior of the whales in nature, hump whales dive around the prey and then start to create bubble in a spiral shaped and swim up toward the surface. This behavior is unique in nature and this maneuver of humpback whales is defined mathematically in order to perform optimization problem.

\section{Mathematical expressions and optimization algorithm}

Here, we presented the principal equations related to the mathematical model used by hump whales for searching the prey and simulate the mechanism of optimization, following three principals rules of encircling the prey, spiral bubble-net maneuver and search for the prey:

\section{Encircling the prey:}

Following Mirjalili, (2016), encircling behavior is mathematically represented by the distance $(d)$, position vectors of the prey $\left(\vec{x}_{\mathrm{p}}\right)$ and humpback whale $\left(\vec{x}_{\mathrm{hw}}\right)$, and using the coefficient vectors and $\vec{A}$ and $\vec{C}$ related to the whales and the prey as showed in the equation (6) and equation (7):

$$
\begin{gathered}
\vec{d}=\left|\vec{C} \cdot \vec{x}_{p}(i)-\vec{x}_{h w}(i)\right| \\
\vec{x}_{h w}(i+1)=\vec{x}_{p}(i)-\vec{A} \cdot \vec{d}
\end{gathered}
$$

Where $i$ is the $i^{\text {th }}$ iteration. The coefficient vectors are determinated as follows in the equation (8) and equation (9), respectively:

$$
\begin{gathered}
\vec{A}=2 \cdot \vec{a} \cdot \overrightarrow{r_{1}}-\vec{a} \\
\vec{C}=2 \cdot \overrightarrow{r_{2}}
\end{gathered}
$$

Where components $\vec{a}$ are lianarly decreased from 2 to 0 over the iterations, and $\vec{r}_{1}$ and $\vec{r}_{2}$ are random vectors in the range $[0,1]$.

In this sense, the search of agents will move around the best solution obtained so far. The humpback whales use the mechanism of bubble-net to attack the prey. This behavior is formulated as follows:

\section{Bubble-net attack mechanism:}

This behavior is grouped in two principal strategies:

i) Shrinking encircling: This mechanism is achieved by decreasing the value of $\vec{a}$ in the equation (8). This value define the strategy for searching $(\vec{A}>1)$ and attacking $(\vec{A}<1)$ the prey.

ii) Spiral updating position: This behavior calculate the distance between the whale and the prey as follows in the equation (10):

$$
\vec{x}_{h w}(t+1)=\vec{d}^{\prime} \cdot e^{b l} \cdot \cos (2 \pi l)+\vec{x}_{p}(t)
$$

Where $\overrightarrow{d^{\prime}}=\left|\vec{x}_{p}(t)-\vec{x}_{h w}(t)\right|$ indicatres the position of ith whale to the prey, $b$ is a constant defining the shape of logarithmic spiral and I is a random number between [-1, $1]$.

To define the quantity of exploration and exploitation if the (WOA) we define a probability of $50 \%$ to choose either the shrinking encircling behavior or the spiral model to update the position of whales during optimization. This mathematical representation for $p<50 \%$ is as follows in equation (11):

$$
\vec{x}_{h w}(\mathrm{t}+1)=\vec{x}_{p}-\vec{A} \cdot \vec{d}
$$

And the representation when $p>50 \%$ is depicted in the equation (12),

$$
\vec{x}_{h w}(t+1)=\overrightarrow{d^{\prime}} \cdot e^{b l} \cdot \cos (2 \pi l)+\vec{x}_{p}(t)
$$

For all synthetic examples we set up the constants $b$ and I as 1 and a random number between $[-1,1]$, respectively. 


\section{Exploration mechanism (Search for prey)}

The mathematical model used for WOA to emphasize exploration is represented as follows in the equation (13):

$$
\vec{d}=\left|\vec{C} \cdot \overrightarrow{X_{\text {rand }}}-\vec{x}_{h w}\right|
$$

Where $\vec{x}_{\mathrm{hw}}$ is expressed by the equation (14)

$$
\vec{x}_{h w}(\mathrm{t}+1)=\overrightarrow{X_{\text {rand }}}-\vec{A} \cdot \vec{d}
$$

Using the same mechanism based on updating the position of whales according to the position of each other, we update the position of the search agent according to randomly chosen search agent instead the best search agent found so far.

\section{Inversion of synthetic data}

We considered layered earth models for testing the effectiveness of WOA. We designed two types of models, (H-type model) and (K-type model). The comparative study is summarized in Table 2 and Table 3 . Schlumberger vertical electrical soundings were generated adding random Gaussian noise of $2 \%$ to direct current resistivity raw data. Initial guesses used for delimiting the lower bound and upper bound of the search space were made based on a qualitative interpretation of the apparent resistivity curves. The Figure 1 and the Figure 2 show a comparison between the true model and the best model obtained using (WOA) for the $\mathrm{H}$ type model and K-type model, respectively. In addition, these Figures include the history of convergence using the WOA.
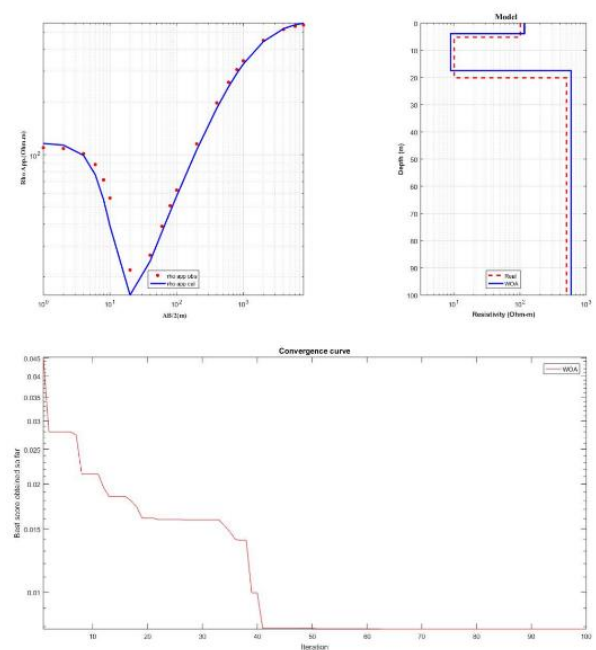

Figure 1 Misfit between observed and calculated data using the best model obtained with WOA. History of convergence using WOA.
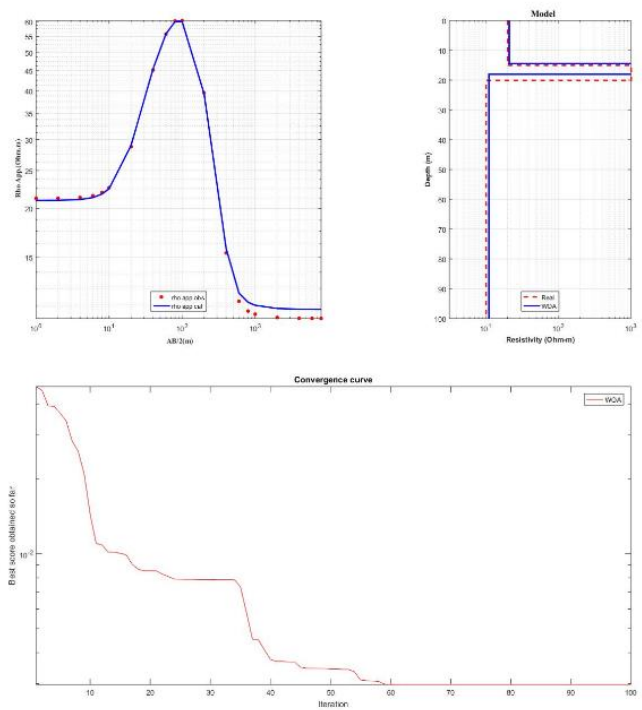

Figure 2 Misfit between observed and calculated data using the best model obtained with WOA. History of convergence using WOA.

The misfit between data and model responses are calculated through the relative error, given as follows in the equation (15):

$$
\text { misfit }=\frac{100}{N} * \sum_{i=1}^{N}\left|\frac{d_{i}-y_{i}}{d_{i}}\right|
$$

All calculated models have misfit values less than $1 \%$. In the other hand, we build a synthetic model corresponding to stratigraphy of Bebedouro Region in Paraná Sedimentary Basin, in Brazil, following a real geoelectric stratigraphy observed in that area (Porsani, et al., 2012.). This stratigraphy is composed by 4 layers: The initial layer with $8 \mathrm{~m}$ of thickness and $200 \Omega . \mathrm{m}$ correspond a layer with unsaturated soil. The second layer composed by a saturated zone is characterized by $25 \Omega . \mathrm{m}$ and $55 \mathrm{~m}$ thick. The resistivity layer below $800 \Omega . \mathrm{m}$ and $500 \mathrm{~m}$ thick is represented by basaltic rock. The last layer correspond to Guarani aquifer with $30 \Omega . \mathrm{m}$, as described in (Bortolozo, et al., 2015). Similar to a two synthetic models presented above, we define the search space based on apparent resistivity curves Table 1 and the results of the inversion process are showed in the Figure 3. The misfit between data and model responses have a misfit less than $0.08 \%$. 


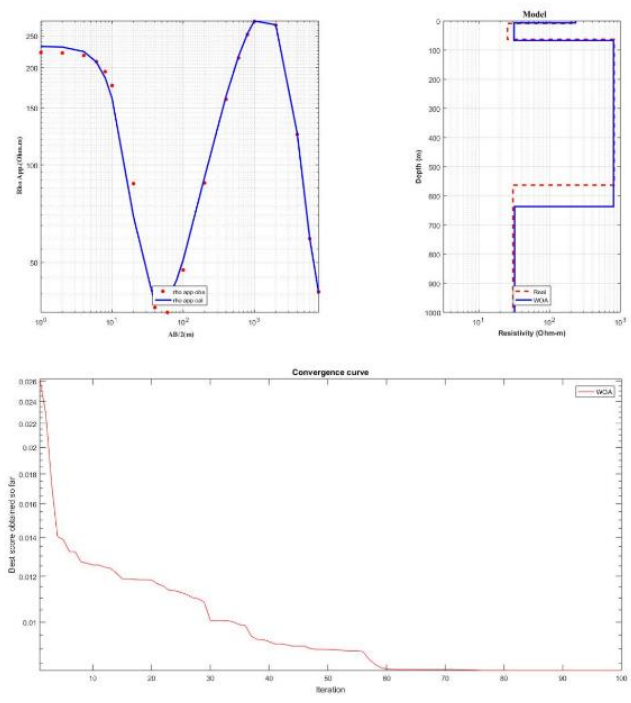

Figure 3 Misfit between observed and calculated data using the best model obtained with WOA. History of convergence using WOA.

\begin{tabular}{|l|l|l|l|}
\hline Parameters & $\begin{array}{l}\text { True } \\
\text { Value }\end{array}$ & Min. & Max. \\
\hline$\rho_{1}(\Omega . \mathrm{m})$ & 100 & 5 & 200 \\
\hline$\rho_{2}(\Omega . \mathrm{m})$ & 10 & 1 & 30 \\
\hline$\rho_{3}(\Omega . \mathrm{m})$ & 500 & 100 & 1000 \\
\hline $\mathrm{h}_{1}(\mathrm{~m})$ & 5 & 0.1 & 10 \\
\hline $\mathrm{h}_{2}(\mathrm{~m})$ & 15 & 1 & 30 \\
\hline
\end{tabular}
he inversion of H-type synthetic data using WOA.

\begin{tabular}{|l|l|l|l|}
\hline Parameters & $\begin{array}{l}\text { True } \\
\text { Value }\end{array}$ & Min. & Max. \\
\hline$\rho_{1}(\Omega . m)$ & 20 & 5 & 120 \\
\hline$\rho_{2}(\Omega . m)$ & 1000 & 50 & 3000 \\
\hline$\rho_{3}(\Omega . m)$ & 10 & 1 & 120 \\
\hline$h_{1}(m)$ & 15 & 2 & 35 \\
\hline$h_{2}(m)$ & 5 & 0.5 & 20 \\
\hline
\end{tabular}

Table 3. True parameters and search space range for the inversion of K-type synthetic data using WOA.

\begin{tabular}{|l|l|l|l|}
\hline Parameters & $\begin{array}{l}\text { True } \\
\text { Value }\end{array}$ & Min. & Max. \\
\hline$\rho_{1}(\Omega . \mathrm{m})$ & 200 & 100 & 300 \\
\hline$\rho_{2}(\Omega . \mathrm{m})$ & 25 & 10 & 50 \\
\hline$\rho_{3}(\Omega . \mathrm{m})$ & 800 & 500 & 1000 \\
\hline$\rho_{4}(\Omega . \mathrm{m})$ & 30 & 10 & 50 \\
\hline $\mathrm{h}_{1}(\mathrm{~m})$ & 8 & 1 & 10 \\
\hline $\mathrm{h}_{2}(\mathrm{~m})$ & 55 & 30 & 60 \\
\hline $\mathrm{h}_{3}(\mathrm{~m})$ & 500 & 100 & 800 \\
\hline
\end{tabular}

. the inversion of Bebedouro Basin using WOA.

\section{Conclusions}

We analyzed the performance of the Whale optimization algorithm (WOA) applied in a set of synthetic data created based on standard curves of resistivity data and a set of synthetic geoelectric data from Paraná Basin in Brazil. We use 7 whales and 100 iterations to achieve a good performance between the true model and the real inverted model with WOA. The misfit of data gives an excellent adjust and offers an additional alternative to obtain among the traditional global optimization methods widely studied in the literature, corresponding to 1D inversion of resistivity data.

\section{Acknowledgments}

We thankful to PROAP founding for providing financial support to participate in this event.

\section{References}

BORTOLOZO, C. A., PORSANI, J. L., DOS SANTOS, F. A. M., \& ALMEIDA, E. R. (2015). VES/TEM 1D joint inversion by using Controlled Random Search (CRS) algorithm. Journal of Applied Geophysics, 112, 157-174.

CHUNDURU, R. K., SEN, M. K., STOFFA, P. L., \& NAGENDRA, R. (1995). Non-linear inversion of resistivity profiling data for some regular geometrical bodies1. Geophysical Prospecting, 43(8), 979-1003.

GHOSH, D. P. (1971). The application of linear filter theory to the direct interpretation of geoelectrical resistivity sounding measurements. Geophysical prospecting, 19(2), 192-217.

GOLDBERG, D. E., \& SASTRY, K. (1989, January). A practical schema theorem for genetic algorithm design and tuning. In Proceedings of the genetic and evolutionary computation conference (Vol. 328335).

KENNEDY, J., \& EBERHART, R. (1995, November). Particle swarm optimization. In Proceedings of ICNN'95-international conference on neural networks (Vol. 4, pp. 1942-1948). IEEE.

KIRKPATRICK, S., GELATT, C. D., \& VECCHI, M. P. (1983). Optimization by simulated annealing. science, 220(4598), 671-680.

KOEFOED, O. (1970). A fast method for determining the layer distribution from the raised kernel function in geoelegtrical 
sounding. Geophysical Prospecting, 18(4), 564-570.

MENKE, W. (1984). Geophysical data analysis: Discrete inverse theory. Academic press.

MIRJALILI, S; LEWIS, A. (2016). The Whale optimization algorithm. Advances in engineering software, 95, 51-67.

PORSANI, J. L., ALMEIDA, E. R., BORTOLOZO, C. A., \& DOS SANTOS, F. A. M. (2012). TDEM survey in an area of seismicity induced by water wells in Paraná sedimentary basin, Northern São Paulo State, Brazil. Journal of applied geophysics, 82, 75-83.

SEN, M. K., \& STOFFA, P. L. (1991). Nonlinear one-dimensional seismic waveform inversion using simulated annealing. Geophysics, 56(10), 1624-1638.

SEN, M. K., BHATTACHARYA, B. B., \& STOFFA, P. L. (1993). Nonlinear inversion of resistivity sounding data. Geophysics, 58(4), 496-507.

TARANTOLA, A. (1987). Inversion of travel times and seismic waveforms. Seismic tomography, 135-157. 